\title{
Partial purification of tightly bound mitochondrial hexokinase from maize (Zea mays L.) root membranes
}

G.L. Rezende', C. Logullo²,

L. Meyer ${ }^{1}$, L.B. Machado ${ }^{1}$, A.L. Oliveira-Carvalho ${ }^{1}$, R.B. Zingali ${ }^{1}$, D. Cifuentes ${ }^{3}$ and A. Galina ${ }^{1}$

\author{
1'Instituto de Bioquímica Médica, Centro de Ciências da Saúde, \\ Universidade Federal do Rio de Janeiro, RJ, Brasil \\ ²Laboratório de Química e Função de Proteínas e Peptídeos, CBB, \\ Universidade Estadual do Norte Fluminense, Campos dos Goytacazes, RJ, Brasil \\ ${ }^{3}$ Departamento de Bioquímica y Biologia Molecular, Universidad de Barcelona, \\ Barcelona, Spain
}

\author{
Correspondence \\ A. Galina \\ Av. Bauhinia, 400 \\ Instituto de Bioquímica Médica \\ UFRJ, CCS, BI. D, sub-solo, Sala 13 \\ 21941.590 Rio de Janeiro, RJ \\ Brasil \\ Fax: +55-21-2270-8647 \\ E-mail: galina@bioqmed.ufrj.br \\ The present address of G.L. Rezende \\ is Laboratório de Fisiologia e \\ Controle de Artrópodes Vetores, \\ Departamento de Entomologia, \\ Instituto Oswaldo Cruz, FIOCRUZ, \\ Manguinhos, Rio de Janeiro, RJ, \\ Brasil. \\ Research supported by CNPq \\ (No. E-26/170.630/2004) and \\ FAPERJ (No. 480378/2005-5).
}

Received December 14, 2005 Accepted May 18, 2006

\begin{abstract}
In mammals, hexokinase (HK) is strategically located at the outer membrane of mitochondria bound to the porin protein. The mitochondrial HK is a crucial modulator of apoptosis and reactive oxygen species generation. In plants, these properties related to HK are unknown. In order to better understand the physiological role of non-cytosolic hexokinase (NC-HK) in plants, we developed a purification strategy here described. Crude extract of $400 \mathrm{~g}$ of maize roots (230 mg protein)

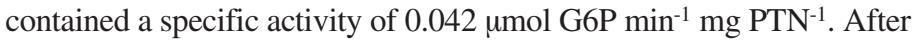
solubilization with detergent two fractions were obtained by DEAE column chromatography, NC-HK 1 (specific activity $=3.6 \mu \mathrm{mol}$ G6P $\mathrm{min}^{-1} \mathrm{mg} \mathrm{PTN}{ }^{-1}$ and protein recovered $=0.7 \mathrm{mg}$ ) and NC-HK 2. A major purification (yield $=500$-fold) was obtained after passage of NC-HK 1 through the hydrophobic phenyl-Sepharose column. The total amount of protein and activity recovered were 0.04 and $18 \%$, respectively. The NCHK 1 binds to the hydrophobic phenyl-Sepharose matrix, as observed for rat brain HK. Mild chymotrypsin digestion did not affect adsorption of NC-HK 1 to the hydrophobic column as it does for rat HK I. In contrast to mammal mitochondrial HK, glucose-6-phosphate, clotrimazole or thiopental did not dissociate NC-HK from maize (Zea mays) or rice (Oryza sativa) mitochondrial membranes. These data show that the interaction between maize or rice NC-HK to mitochondria differs from that reported in mammals, where the mitochondrial enzyme can be displaced by modulators or pharmacological agents known to interfere with the enzyme binding properties with the mitochondrial porin protein.
\end{abstract}

\section{Introduction}

Hexokinase (HK) [EC 2.7.1.1] catalyzes the crucial step in hexose metabolism which converts free hexose to hexose-6-phosphate, using ATP. Since the 1950's, the existence of particulate HK isoforms has been observed in
Key words

- Hydrophobic interaction

chromatography

- Maize

- Mitochondrial hexokinase

- Non-cytosolic hexokinase

- Purification plants (1-4). The association of HK with the outer mitochondrial membrane (5-11), the outer envelope membrane of plastids $(12,13)$ or with the Golgi apparatus (14) has been reported. Maize (Zea mays) root has a non-cytosolic hexokinase (NC-HK) bound to mitochondria and Golgi vesicles. These HK activities 
are inhibited by micromolar concentrations of ADP and the hexose analogues glucosamine, mannoheptulose and $\mathrm{N}$-acetylglucosamine, which are markers of HKs involved in the sugar sensing signaling cascade in plants or in uridine diphosphoglucose formation in maize roots $(11,14-17)$. Sugar sensing is an important event in plant metabolism, in which the pattern of genetic expression of a tissue is altered by the presence of free hexoses (18). Interestingly, in maize roots the cytosolic hexokinase and fructokinase isoforms are not inhibited by ADP or hexose analogues, suggesting that NC-HKs are the main isoforms involved in this specific hexose signaling pathway (14). The sugar sensing pathways have not been established. However, glucose-insensitive and glucose-oversensitive mutants have revealed extensive and intimate connections between glucose and plant hormone signaling pathways such as ethylene and abscisic acid (18, 19). Accordingly, in Arabidopsis thaliana, AtHK 1 activity is found associated with mitochondria, similarly to the NC-HK activity found in maize (Zea mays) or rice (Oryza sativa) roots that also exhibit the sugar sensing response $(14-16,18,19)$. The HK sensing function may depend on its subcellular localization, translocation and/or interactions with downstream effectors, but these properties are still unknown. It has been suggested that in castor bean (Ricinus communis) $\mathrm{HK}$ and mitochondria interact in a way similar to that described for rat brain $(6,20)$. In the rat brain, $\mathrm{HK}$ is associated with mitochondria through the porin protein, also called voltage-dependent anion channel (21). The rat HK dissociates from the mitochondrial porin in a reversible manner depending on the levels of glucose-6-phosphate (22-24). Additionally, pharmacological drugs such as the fungicide clotrimazole and the anesthetic thiopental can also dissociate mammalian HK from the porin, releasing the enzyme from mitochondria $(25,26)$. The ability of these drugs to dissociate HK from plant mitochondria has not been tested. It was shown that the first $10 \mathrm{~N}$-terminal residues of rat HK are responsible for its association with porin (21). Mild chymotrypsin digestion removes the first $10 \mathrm{~N}$-terminal residues from the rat brain $\mathrm{HK}$ which, without these $\mathrm{N}$-terminal residues, loses the ability to reattach to its mitochondrial receptor and also to be adsorbed to hydrophobic resins such as phenyl-Sepharose (27). On the other hand, it was shown that in spinach (Spinacia oleracea) leaves, SoHK 1 interacts with the outer envelope membrane of chloroplasts by its $24 \mathrm{~N}$-terminal residues, and has been suggested to function as a membrane anchor. This observation is supported by the fact that truncated spinach $\mathrm{SoHK}$ 1 without $24 \mathrm{~N}$-terminal residues does not associate with the outer chloroplast envelope membrane (13). Wiese et al. (13) suggested that this hydrophobic $\mathrm{N}$-terminal is immersed directly into the membrane independently of the presence of any receptor protein. Similar to spinach $\mathrm{SoHK}$, it was found that the rice OsHK 1 gene product is associated with the mitochondrial membrane and also presents the first 24 hydrophobic N-terminal residues (28). The DNA sequence of rice OsHK 1 (GenBank accession No. AF372831) is very closely related to the maize HK sequence found in GenBank (accession No. AY125979), suggesting that maize HK may bind to mitochondria in a way similar to that observed for rice HK. Apart from an R. communis study (6), no further investigation concerning the properties of interaction of HK with mitochondria was performed so far in plants. In an attempt to gain insights about the nature of NC-HK association with maize mitochondria, the separation of this enzyme from plant organelles is essential.

Thus, the aims of the present investigation were: 1) to study the interaction of maize NC-HK and mitochondria and compare it with that of $\mathrm{HK}$ and mitochondria observed in the rat brain, and 2) to establish a procedure for the purification of a mito- 
chondrial membrane-bound HK. In addition, to study the sensitivity of plant HKs to modulators of the binding of HK to mitochondria used in mammals.

\section{Material and Methods}

Isolation of mitochondria from rice, maize root, and rat brain

In order to isolate rice root mitochondria containing large amounts of NC-HK (named OsHK 1), we used essentially the same protocol as described for maize mitochondria isolation. Maize roots ( $400 \mathrm{~g}$ ) were obtained as described before (14) and were homogenized in two volumes of cold buffer A (0.1 $\mathrm{M}$ Tris- $\mathrm{HCl}, \mathrm{pH} 8.0,10 \%$ glycerol, $0.5 \%$ PVP-40, 3.3 mM DTT, $10 \mathrm{mM}$ glucose, 1.3 $\mathrm{mg} / \mathrm{mL}$ BSA, $0.15 \mathrm{M} \mathrm{KCl}, 1 \mathrm{mM} \mathrm{PMSF}$, and 5 mM EDTA). The crude mitochondrial pellet was obtained from the $10,000 \mathrm{~g}$ differential centrifugation. The mitochondria were isolated as described before (11) and were re-suspended in $35 \mathrm{~mL}$ of cold buffer B (20 mM HEPES-Tris, $\mathrm{pH}$ 8.0, 0.3 M mannitol, 4 mM EGTA, 4 mM EDTA, 0.2 mM PMSF, 4 $\mathrm{mM}$ B-mercaptoethanol, $0.1 \mathrm{M} \mathrm{KCl}$, and 10 $\mathrm{mM}$ glucose). Briefly, the pellet was washed three times with $40 \mathrm{~mL}$ of buffer $\mathrm{B}$ and centrifuged at $7000 \mathrm{~g}$ for $15 \mathrm{~min}$ at $4^{\circ} \mathrm{C}$. The final pellet was re-suspended in buffer $\mathrm{B}$ to a final concentration of 10 to $20 \mathrm{mg}$ protein/ $\mathrm{mL}$ and stored in liquid nitrogen.

Rat brain mitochondria were isolated as described (22) in a buffer containing $10 \mathrm{mM}$ Tris-HCl, pH 7.4, 0.25 M sucrose, $0.2 \mathrm{mM}$ PMSF, $0.2 \mathrm{mM}$ EDTA, and $10 \mathrm{mM}$ glucose. The mitochondrial pellet was re-suspended to a final concentration of $20 \mathrm{mg}$ protein $/ \mathrm{mL}$ and used immediately.

\section{Hexokinase activity assay}

Each fraction was assayed for HK activity as previously described (29) in $20 \mathrm{mM}$ Tris-HCl, pH 7.5, 6 mM MgCl 2,2 mM glu- cose, $1 \mathrm{mM}$ ATP, $2 \mathrm{mM}$ PEP, $0.1 \%$ (v/v) Triton X-100, $10 \mathrm{mM} \mathrm{NaN}_{3}, 6$ units/mL pyruvate kinase, and protein to a final concentration of 5 to $50 \mu \mathrm{g} / \mathrm{mL}$. The reaction was started by adding protein fractions and was stopped after 5 to $10 \mathrm{~min}$ at $35^{\circ} \mathrm{C}$ by heating for $1 \mathrm{~min}$ at $100^{\circ} \mathrm{C}$. The glucose-6phosphate formed was measured by adding an equal volume of a solution containing 20 $\mathrm{mM}$ Tris-HCl, $\mathrm{pH} 7.5,6 \mathrm{mM} \mathrm{MgCl}_{2}, 1$ unit/ mL glucose-6-phosphate dehydrogenase ( $L e u$ conostoc mesenteroides; Sigma, St. Louis, $\mathrm{MO}$, USA), and $0.3 \mathrm{mM} B-\mathrm{NAD}^{+}$. Absorbance was read at $340 \mathrm{~nm}$ using a molar extinction coefficient of $6.22 \mathrm{M}^{-1}$. In all cases, activities were linear up to $30 \mathrm{~min}$ and linear with the amount of extract added. Alternatively, the HK activity was measured in a 96-well microplate reader in a final volume of $0.15 \mathrm{~mL}$. The yeast and rat $\mathrm{HK}$ activities were determined with the microplate reader, as described above but in a medium containing $0.6 \mathrm{mM} \beta \mathrm{NADP}^{+}$instead of $\mathrm{B}-\mathrm{NAD}^{+}$. The reactions were started with ATP at a final concentration of $1 \mathrm{mM}$.

Solubilization of hexokinase from rice, maize, or rat mitochondria

The maize, rice roots or rat brain mitochondria with final protein concentration of $0.5 \mathrm{mg} / \mathrm{mL}$ were incubated for $30 \mathrm{~min}$ at $28^{\circ} \mathrm{C}$ in a medium containing $10 \mathrm{mM}$ Tris- $\mathrm{HCl}, \mathrm{pH}$ 7.5, $2 \mathrm{mM}$ ß-mercaptoethanol, with glucose6-phosphate at final concentrations of 0 to 5 $\mathrm{mM}$. Rice or rat mitochondria were also incubated with $10 \mu \mathrm{M}$ clotrimazole, $2 \mathrm{mM}$ thiopental and $5 \mathrm{mM}$ glucose-6-phosphate and the maize mitochondria were also incubated with $150 \mathrm{mM}$ glucose, $150 \mathrm{mM}$ fructose, $5 \mathrm{mM}$ mannose-6-phosphate, $5 \mathrm{mM}$ UDP, $5 \mathrm{mM}$ UDP-glucose, $10 \mu \mathrm{M}$ clotrimazole, $2 \mathrm{mM}$ thiopental, or $5 \mathrm{mM}$ glucose-6-phosphate. The maize, rice or rat mitochondrial $\mathrm{HK}$ was solubilized with detergents. Maize, rice roots or rat brain mitochondria with final protein concentration of $1 \mathrm{mg} / \mathrm{mL}$ were incubated for $30 \mathrm{~min}$ 
at $4^{\circ} \mathrm{C}$ in a medium containing $15 \mathrm{mM}$ Tris$\mathrm{HCl}, \mathrm{pH}$ 7.5, 4 mM B-mercaptoethanol, 10 mM glucose, 1 mM EDTA, 1 mM PMSF, 0.2 $\mathrm{M} \mathrm{KCl}$, and $1 \%$ Triton X-100 (v/v). Alternatively, maize NC-HK was solubilized with CHAPS at different final concentrations ranging from 0 to $6 \%(\mathrm{w} / \mathrm{v})$. Mitochondria from maize, rice or rat were then centrifuged at $105,000 \mathrm{~g}$ for $45 \mathrm{~min}$ at $4^{\circ} \mathrm{C}$. The supernatants were collected and the mitochondrial pellets were re-suspended in the same medium but without the solubilizing compounds. A $10-\mu \mathrm{L}$ aliquot from each supernatant and pellet was used to assay HK activity in the microplate reader system, but with $1.5 \mathrm{mM} B-\mathrm{NAD}^{+}$or $\beta-$ $\mathrm{NADP}^{+}$in the glucose-6-phosphate experiments. The percentage of solubilized (released) HK activity was calculated as the ratio (activity in supernatant)/(activity in supernatant + activity in pellet).

\section{DEAE-Toyopearl chromatography}

The mitochondrial pellet from maize roots was incubated with $4 \%$ CHAPS as described above in a total volume of $13 \mathrm{~mL}$. The solubilized HK activity was diluted five times in buffer D (10 mM Tris-HCl, pH 8.4, 10 $\mathrm{mM}$ glucose, $4 \mathrm{mM}$ ß-mercaptoethanol, 0.2 mM EDTA, and $0.2 \mathrm{mM} \mathrm{PMSF}$ ) or 20 units of yeast HK (Sigma type C-300) were added to the buffer $\mathrm{D}$, when necessary for the binding studies (Figure 3), and was applied to a DEAE-Toyopearl column $(1.7 \times 8.2 \mathrm{~cm})$ pre-equilibrated with buffer $\mathrm{D}$. The column was washed with buffer D until effluent protein reached near zero and a $100-\mathrm{mL}$ linear gradient of 0 to $0.4 \mathrm{M} \mathrm{NaCl}$ in the same buffer was then applied to the column. Fractions of $7.5 \mathrm{~mL} /$ tube were collected before the gradient was applied and fractions of $2.5 \mathrm{~mL} /$ tube were collected during the gradient. Protein elution was monitored at $280 \mathrm{~nm}$. The HK activity in each fraction was assayed in the microplate reader. Two activity peaks, named NC-HK 1 and NC-HK 2 , were observed. The fractions containing activity from NC-HK 1 or NC-HK 2 were pooled together in three tubes of $2.5 \mathrm{~mL}$ each and stored at $-80^{\circ} \mathrm{C}$. The run was performed at $4^{\circ} \mathrm{C}$ at a flow rate of $2 \mathrm{~mL} / \mathrm{min}$.

\section{Chromatography on phenyl-Sepharose}

Aliquots of NC-HK 1, NC-HK 2 or yeast HK (Sigma type C-300) from the DEAE column were diluted 4.4 times in buffer $\mathrm{F}$ (15 mM Tris/HCl, pH 7.5, 10 mM glucose, 4 mM ß-mercaptoethanol, 0.2 mM EDTA, 2 mM PMSF, $1.5 \mathrm{M} \mathrm{NaCl}$ ) and were applied to a phenyl-Sepharose column $(1.7 \times 6 \mathrm{~cm})$ pre-equilibrated with buffer F. For the NCHK 1 fraction, the total amount of protein applied was $0.73 \mathrm{mg}$ and the total activity was $2.64 \mu \mathrm{mol}$ glucose-6-phosphate/min. For the NC-HK 2 fraction, the total amount of protein applied was $1.04 \mathrm{mg}$ and the total activity was $0.70 \mu \mathrm{mol}$ glucose-6-phosphate/ min. The column was washed with buffer $\mathrm{F}$ until protein in the effluent reached near zero and a 52-mL linear gradient of 0 to $100 \%$ buffer $\mathrm{G}$ (buffer $\mathrm{F}$ without $\mathrm{NaCl}$ ) was then applied to the column. Next, the column was washed with $56 \mathrm{~mL}$ of buffer G. Fractions of $8 \mathrm{~mL} /$ tube were collected before the gradient and fractions of $4 \mathrm{~mL} /$ tube were collected after the gradient. Protein elution was monitored at $280 \mathrm{~nm}$. The fractions containing activity of NC-HK 1 or NC$\mathrm{HK} 2$ were pooled and stored at $-80^{\circ} \mathrm{C}$. Soluble yeast $\mathrm{HK}$, which is cytosolic, was applied to a phenyl-Sepharose column as a control. Fractions with activity from the yeast HK, previously loaded onto a DEAE column, were pooled and applied to a phenyl-Sepharose column as described above except that, after the gradient, the column was washed with 32 $\mathrm{mL}$ of buffer $\mathrm{G}$ and fractions of $4 \mathrm{~mL} /$ tube were collected throughout the run. The fractions with activity from the rat brain mitochondrial HK, previously solubilized with glucose-6-phosphate and loaded onto a DEAE column, were pooled and applied to a phenylSepharose column as described above except 
that, after the gradient, the column was washed with $48 \mathrm{~mL}$ of buffer $\mathrm{G}$. The HK activity in the fractions of each run was assayed with the microplate reader. All runs were performed at $4^{\circ} \mathrm{C}$ at a flow rate of $1 \mathrm{~mL} / \mathrm{min}$.

\section{Mild chymotrypsin digestion of hexokinases}

Samples from maize or rat HKs eluted from the DEAE column were incubated in a digestion medium adapted from Kurokawa et al. (27), containing $20 \mathrm{mM}$ Tris/ $\mathrm{HCl}, \mathrm{pH} 7.5,2$ mM EDTA, 5 mM ß-mercaptoethanol, 10 $\mathrm{mM}$ glucose and chymotrypsin at a concentration ranging from 0.5 to $1.2 \mu \mathrm{g} / \mathrm{mL}$, depending on the $\mathrm{HK}$ utilized. The digestions were performed at $30^{\circ} \mathrm{C}$ for $20 \mathrm{~min}$ and were stopped by adding $2.2 \mathrm{mM}$ PMSF. The treated samples were then loaded onto the phenyl-Sepharose column as described above. Mild chymotrypsin digestion resulted in a 25 to $33 \%$ loss of the initial $\mathrm{HK}$ activities measured.

\section{Superose 12 chromatography}

Samples of NC-HK 1 eluted from the phenyl-Sepharose column were concentrated in a Speed Vac apparatus and loaded onto a Superose 12 H/R column (Amersham, Pharmacia Biotech, Waukesha, WI, USA) equilibrated with a buffer containing $15 \mathrm{mM}$ Tris$\mathrm{HCl}, \mathrm{pH} 7.5,8 \mathrm{mM}$ glucose, and $0.5 \mathrm{M}$ $\mathrm{NaCl}$, at a flow rate of $0.5 \mathrm{~mL} / \mathrm{min}$. Fortytwo fractions of $0.5 \mathrm{~mL}$ were collected. The HK activity of each fraction was assayed with the microplate reader and protein elution was monitored at $214 \mathrm{~nm}$. The standard proteins used for $\mathrm{Mr}$ calibration were bovine serum albumin $(66,000)$, ovalbumin $(45,000)$ and cytochrome $c(12,000)$.

\section{Thermal stability of non-cytosolic hexokinase 1}

Samples of NC-HK 1 eluted from the DEAE column were incubated at $50^{\circ} \mathrm{C}$ for 85 min or at $-80^{\circ} \mathrm{C}$ for 13 days and aliquots were taken at different times for HK activity determination with the microplate reader, as described earlier.

\section{Protein determination}

Protein determination was performed by the method of Lowry et al. (30). When the protein concentration was too low, or when there was buffer interference, the samples were precipitated with $7 \%$ TCA (w/v) on ice for $5 \mathrm{~min}$, followed by centrifugation at $20,000 \mathrm{~g}$ for $15 \mathrm{~min}$ at $4^{\circ} \mathrm{C}$ and the proteins were re-suspended in $0.08 \mathrm{~mL}$ with $0.1 \%$ SDS (w/v) before carrying out the protein determination according to the method of Lowry et al. (30). Bovine serum albumin was used as standard.

\section{Results}

\section{Solubilization of mitochondrial hexokinases}

Glucose-6-phosphate, at $5 \mathrm{mM}$, was efficient to dissociate HK from rat brain $(85 \%)$ and $R$. communis developing seed $(100 \%)$ (6) mitochondria but not from $O$. sativa or $Z$. mays root mitochondria (data not shown). Accordingly, under our experimental conditions, glucose-6-phosphate released more than $80 \%$ HK from rat brain mitochondria (Figure 1A, filled squares). The same treatment done with glucose-6-phosphate or with different sugar metabolites found in plant cells (150 mM glucose or fructose; $5 \mathrm{mM}$ glucose-6-phosphate, mannose-6-phosphate, UDP or UDP-glucose) was unable to release NC-HK from maize mitochondria (Figure 1A, open circles, and data not shown). For rice root mitochondria, glucose-6-phosphate was also ineffective to release NC-HK. The pharmacological agents clotrimazole, at 10 $\mu \mathrm{M}$, or thiopental, at $2 \mathrm{mM}$, which are able to solubilize HK from mammalian mitochondria $(25,26)$, were also able to release HK from rat brain mitochondria, but not the NC$\mathrm{HK}$ from maize or rice root mitochondria 
(data not shown). Solubilization of mitochondrial HK activity from all species was attained only with $1 \%(\mathrm{v} / \mathrm{v})$ Triton X-100 detergent. CHAPS at $4 \%(w / v)$ (Figure 1B) was also effective in solubilizing the maize enzyme by $90 \%$ and this detergent was used for NC-HK isolation in order to avoid interference with further purification steps $(31,32)$.
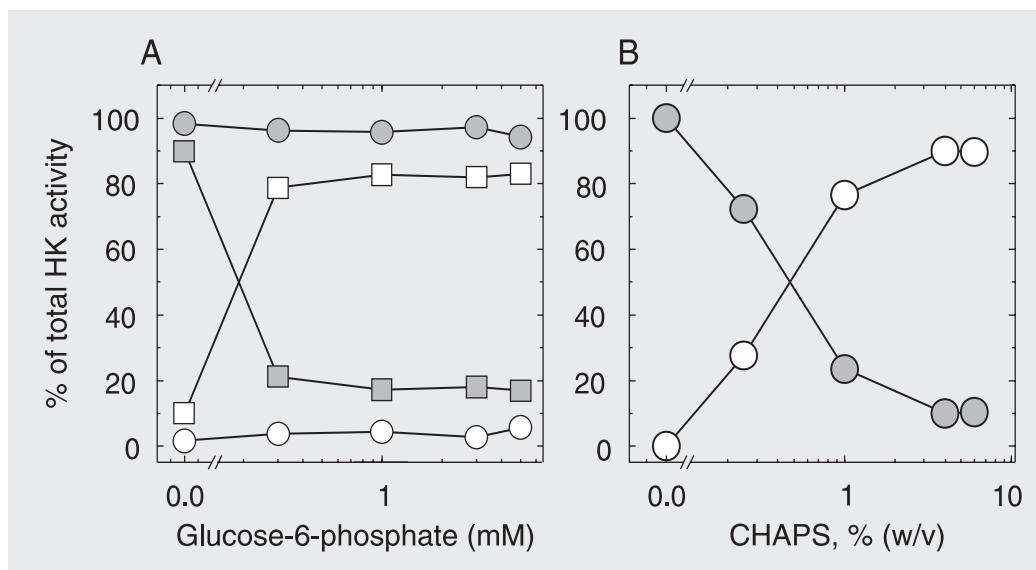

Figure 1. Solubilization of mitochondrial hexokinase (HK) activity with glucose-6-phosphate and CHAPS. A, Rat brain HK (squares) and maize roots HK (circles) from mitochondria incubated with glucose-6-phosphate. B, Maize root mitochondria incubated with CHAPS (\% as $w / v)$. The total HK activity is the sum of the supernatant (open symbols) and the pellet (filled symbols). The figure presents representative experiments from six mitochondrial preparations.

\section{Non-cytosolic hexokinase purification}

A summary of NC-HK purification from maize root mitochondria is presented in Table 1. Since the enzymatic activity is most stable in $10 \mathrm{mM}$ glucose (Table 2), all buffers used during the purification contained $10 \mathrm{mM}$ glucose. After passage through DEAE-Toyopearl, the NC-HK activity was separated into two components designated NC-HK 1 and NC-HK 2 (Figure 2A), as reported previously (29). The first three steps increased the specific activity of NC-HK 1 by 18.5 fold, but the phenyl-Sepharose hydrophobic interaction chromatography step (Figure 2B) resulted in a 488-fold increase in the specific activity of NC-HK 1 in an 18\% yield compared to the crude extract pool (Table 1). However, the purification factor and yield observed for NC-HK 2 after phenyl-Sepharose chromatography were much lower, i.e., 21.6 and $2.2 \%$, respectively (Table 1 ).

Since the NC-HK 2 activity could not be isolated in sufficient quantity, only the NCHK 1 activity was used for further experiments. An attempt was made to further purify NC-HK 1 by gel filtration on a Superose 12 column. Although it was possible to sepa-

Table 1. Purification of non-cytosolic hexokinases from maize roots.

\begin{tabular}{|c|c|c|c|c|c|c|}
\hline \multirow[t]{2}{*}{ Step } & \multirow{2}{*}{$\begin{array}{l}\text { Volume } \\
\text { (mL) }\end{array}$} & \multirow{2}{*}{$\begin{array}{l}\text { Protein } \\
\text { (mg) }\end{array}$} & \multicolumn{2}{|c|}{ Activity } & \multirow{2}{*}{$\begin{array}{l}\text { Purification } \\
\text { activity }\end{array}$} & \multirow{2}{*}{$\begin{array}{c}\text { Recovery } \\
(\%)\end{array}$} \\
\hline & & & Total & Specific $^{+}$ & & \\
\hline Crude extract & 282 & 231 & $9.71 \pm 0.85$ & $0.042 \pm 0.06$ & 1 & 100 \\
\hline Mitochondrial pellet ${ }^{++}$ & 0.86 & 12.6 & $6.46 \pm 0.58$ & $0.514 \pm 0.05$ & 12.2 & 66.5 \\
\hline CHAPS solubilization & 12.4 & 5.33 & $6.32 \pm 0.42$ & $1.185 \pm 0.4$ & 28.2 & 65 \\
\hline NC-HK 1 after DEAE & 18.5 & 0.73 & $2.64 \pm 1.1$ & $3.6 \pm 0.5$ & 85.7 & 27.2 \\
\hline NC-HK 1 after phenyl-Sepharose column chromatography & 31.4 & 0.09 & $1.77 \pm 0.3$ & $20.5 \pm 2.5$ & 488 & 18.2 \\
\hline NC-HK 2 after DEAE & 20.5 & 1.04 & $0.70 \pm 0.4$ & $0.674 \pm 0.6$ & 16.0 & 7.3 \\
\hline NC-HK 2 after phenyl-Sepharose column chromatography & 23.5 & 0.24 & $0.22 \pm 0.1$ & $0.909 \pm 0.3$ & 21.6 & 2.2 \\
\hline
\end{tabular}

Enzyme activity was measured at $35^{\circ} \mathrm{C}$ as described in the first assay system in the Material and Methods section. The protein of each step was measured by the method of Lowry et al. (30).

${ }^{+}$Specific activity was calculated dividing the total activity recovered in the fraction by the total amount of protein recovered in that fraction. The specific activity is reported as $\mu \mathrm{mol}$ G6P min ${ }^{-1} \mathrm{mg} \mathrm{PTN}^{-1}$. Data are reported as means \pm SEM. The NC-HK activity of the total homogenate was calculated as the difference between the specific activity of the total homogenate and the specific activity of the supernatant of the $10,000 \mathrm{~g}$ centrifugation. ${ }^{++}$The mitochondrial pellet corresponds to the pellet of the $10,000 \mathrm{~g}$ centrifugation, washed three times. The table shows representative experiments. G6P = glucose-6-phosphate; NC-HK = non-cytosolic hexokinase. Purification factor is the ratio of the specific activity of a fraction divided by the specific activity of the crude extract. 
rate NC-HK 1 from other contaminating proteins of higher and lower molecular weight (Figure 2C), it was not possible to calculate the yield and purification fold of this step due to very small protein recovery at this stage. In addition, due to the small quantity of the protein, SDS-PAGE analysis was not possible. According to elution position, NCHK 1 has an apparent molecular weight of 38,000 (Figure 2C).

\section{Sensitivity to inhibitors and thermal stability of non-cytosolic hexokinase 1}

NC-HK from maize is non-competitively inhibited by its reaction product ADP and competitively inhibited by the sugar analogue mannoheptulose $(11,14,29)$. The $\mathrm{IC}_{50}$ for the inhibition of purified NC-HK 1 (after phenyl-Sepharose chromatography) was essentially the same as for ADP $(70 \pm 21 \mu \mathrm{M}$, mean \pm SEM) and mannoheptulose (450 \pm

Figure 2. A, Anion exchange chromatography on a DEAE-Toyopearl column of the CHAPS-solubilized activity of NC-HK from mitochondrial maize roots. HK activity (circles). The profile of protein elution was monitored at $280 \mathrm{~nm}$ (lozenges), and an $\mathrm{NaCl}$ gradient from 0 to $0.4 \mathrm{M}$ (broken line) was used. The presence of the two isoforms of NC-HK (NC-HK 1 and NC-HK 2) is indicated. The figure illustrates a representative experiment of nine mitochondrial preparations. The recovered NC-HK 2 activity corresponded to $33.6 \pm 5.1 \%$ (mean $\pm \mathrm{SEM}$ ) of the total NC-HK activity. $B$, Hydrophobic interaction of NC-HK 1 chromatography on a phenyl-Sepharose column. NC-HK 1 (circles) was eluted from the DEAE column, the order of elution was monitored at $280 \mathrm{~nm}$ (lozenges), and an $\mathrm{NaCl}$ gradient from 1.5 to zero $M$ (broken line) was used. The figure shows a representative experiment of six mitochondrial preparations. $C$, Superose 12 gel filtration chromatography of NC-HK 1 activity after elution from the phenylSepharose column. NC-HK 1 activity (circles) was measured as described in Material and Methods. The profile of protein elution was monitored at $214 \mathrm{~nm}$ (lozenges). The arrows indicate the standards of native molecular weight markers of bovine serum albumin (66 kDa), NC-HK 1 (38 kDa) and cytochrome C (12 kDa). The figure shows a representative experiment of four mitochondrial preparations. NC-HK $=$ non-cytosolic hexokinase.
$31 \mu \mathrm{M})$ when NC-HK was bound to mitochondria (data not shown). The stability of NC-HK 1 activity after DEAE-Toyopearl chromatography was determined at $50^{\circ} \mathrm{C}$ for

Table 2. Thermal and storage stability of NC-HK 1 activity from the DEAE column.

\begin{tabular}{lcc}
\hline & \multicolumn{2}{c}{$\%$ remaining } \\
& NC-HK 1 activity \\
\cline { 2 - 3 } & +Glucose & -Glucose \\
& & \\
\hline Thermal stability at $50^{\circ} \mathrm{C}$ & 100 & 35 \\
35 min & 89 & 13 \\
85 min & & \\
Storage stability at $-80^{\circ} \mathrm{C}$ & 100 & 75 \\
4 days & 99 & 32 \\
13 days & & \\
\hline
\end{tabular}

Aliquots were removed at the different times and hexokinase activity was measured with a microplate reader. The table shows representative experiments of three DEAE preparations. The glucose concentration was $10 \mathrm{mM}$. NC-HK = noncytosolic hexokinase.
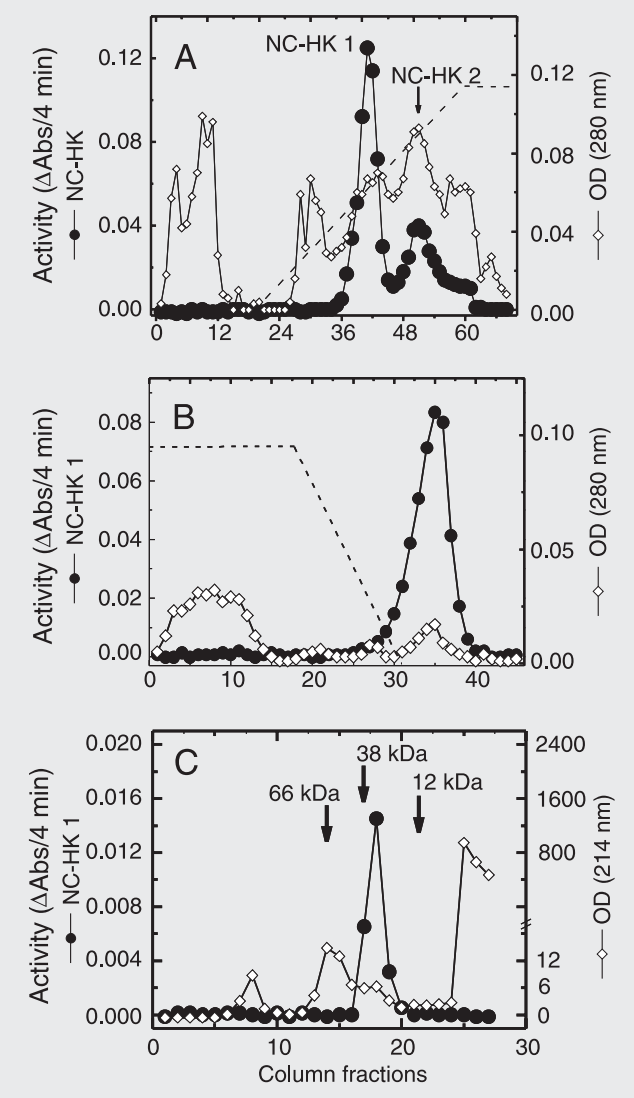
Figure 3. Effect of limited chymotrypsin hydrolysis on hydrophobic interaction chromatographic behavior of maize, rat and yeast hexokinases. $A$, NC-HK 1 eluted from the DEAE column (Figure 2A) with (open circles) and without (filled circles) chymotrypsin digestion; $B$, rat brain $\mathrm{HK}$ activity eluted from the DEAE column with (open squares) and without (filled squares) chymotrypsin digestion; $C$, yeast HK activity not exposed to chymotrypsin hydrolysis eluted from the DEAE column (triangles). $\mathrm{NaCl}$ gradient from 1.5 to $0 \mathrm{M}$ (broken lines). The figure shows a representative experiment of four mitochondrial preparations. $\mathrm{NC}-\mathrm{HK}=$ non-cytosolic hexokinase.
85 min and at $-80^{\circ} \mathrm{C}$ for 13 days (Table 2). After phenyl-Sepharose chromatography, similar stability characteristics were found for NC-HK 1 (data not shown). The presence of $10 \mathrm{mM}$ glucose was essential to stabilize the enzyme (Table 2).

\section{Effect of limited chymotrypsin hydrolysis} on hydrophobic interactions of maize with cytosolic yeast and rat brain mitochondrial hexokinases

The elution profiles from a Sepharose hydrophobic column of a cytosolic yeast or glucose-6-phosphate-solubilized mitochondrial rat brain HKs and NC-HK 1 purified from maize were compared before and after chymotrypsin hydrolysis (Figure 3 ). The elution profile of NC-HK 1 applied to the hydrophobic Sepharose column is shown in Figure 3A (filled circles). The maize enzyme has a strong hydrophobic nature, since it was tightly adsorbed to the column, where

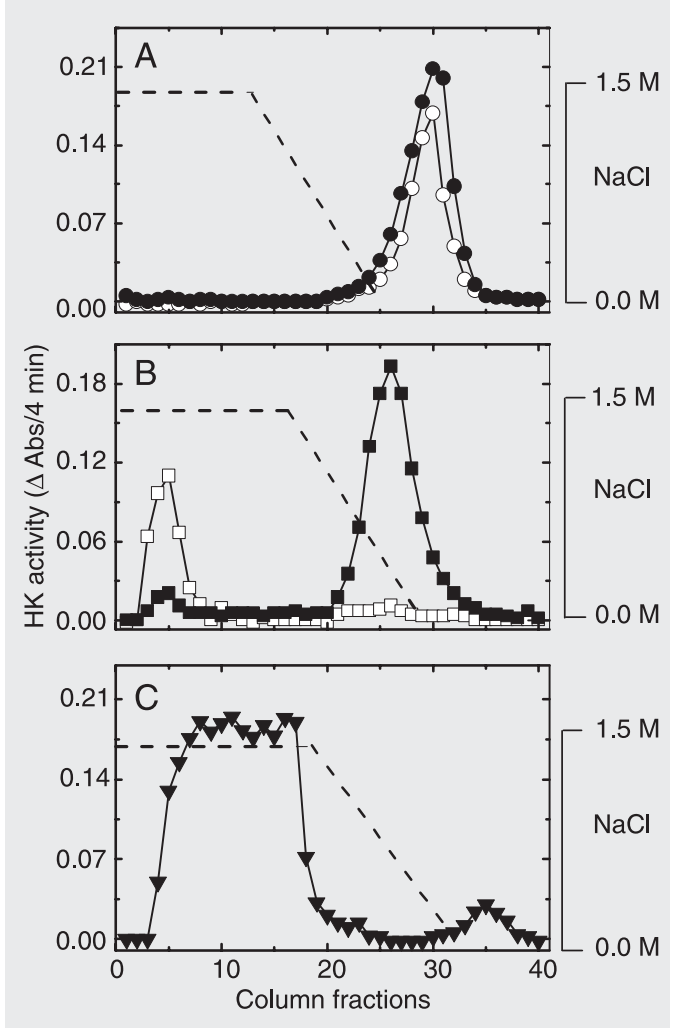

$90 \%$ of total activity was eluted after the gradient achieved $0 \mathrm{M} \mathrm{NaCl}$ concentration (fractions 25 to 33, Figure 3A). Chymotrypsin hydrolysis did not modify the interaction with the resin (Figure 3A, open circles). Rat brain HK was also adsorbed to this column (Figure 3B, filled squares), but after chymotrypsin hydrolysis (Figure 3B, open squares) the enzyme was not retained by the resin. Yeast HK was not adsorbed to the phenylSepharose column (Figure 3C, triangles), as expected for a cytosolic HK (27).

\section{Discussion}

\section{Purification of non-cytosolic hexokinase from maize roots}

NC-HK 1 was purified 488-fold from maize roots by a 4-step method with $18.2 \%$ recovery (Table 1). The extent of purification achieved for this NC-HK was higher than that reported for other HKs from different plant species and different tissues (3336). Different from studies on cytosolic enzymes, phenyl-Sepharose step was 50 times more efficient in purifying maize NC-HK 1 with similar yields (Table 1), indicating that this is a suitable step for the purification of NC-HKs. Several studies have been performed with non-cytosolic HKs from different plants $(1-3,5-13,28,37)$. However, since these studies focused on the sub-cellular localization or the determination of the kinetic properties of these enzymes, the current study presents for the first time a procedure for the purification of particulate HKs from plants.

The apparent molecular weight of NCHK 1, estimated to be 38,000 by gel filtration chromatography (Figure 2C), is similar to those of other hexose kinases found in prokaryotes (38), also determined by gel filtration chromatography, cytosolic HK isoforms of castor bean, barley leaves, maize endosperm, and potatoes $(6,8,33,34)$ and two particulate HK activities detected, but not 
purified, in castor bean (6).

Additional chemical characterization of NC-HK 1 is difficult due to the large amount of maize seedlings (more than $400 \mathrm{~g}$ ) required to obtain less than $90 \mu \mathrm{g}$ of protein in the phenyl-Sepharose step (Table 1). The NC-HK 2 was partially purified but the method used was ineffective for the purification of activity, which was enriched only 21.6-fold (Table 1).

\section{Association between hexokinase and mitochondria in plants and mammals. Are there two distinct types of interaction?}

The present results indicate that NC-HKs from maize and rice roots have unique characteristics regarding the nature of their interaction with mitochondria when compared to the mechanism described for castor bean and rat brain. For NC-HK from maize and rice, it is suggested a stronger hydrophobic character and this property may be potentially important to promote a tight association between $\mathrm{HK}$ and mitochondria in maize. The rat brain HK possesses a site of cleavage for chymotrypsin on its hydrophobic N-terminal $(20,21,23,24,27,38)$. Mild chymotrypsin digestion effectively removes the $\mathrm{N}$-terminal from rat $\mathrm{HK}$, preventing the enzyme from associating with mitochondria or adhering to the phenyl-Sepharose column (27). However, this digestion did not alter the elution profile of NC-HK 1 on the phenylSepharose column (Figure 3A and B, open symbols), showing that maize NC-HK 1 is not able to detach from the column under the same conditions of treatment. The mild digestion effectively reduced NC-HK 1 activity, with a $25 \%$ reduction in total activity (Figure 3A, filled and open circles). This indicates that chymotrypsin digestion did in fact hydrolyze NC-HK 1, but was unable to remove any essential hydrophobic domains involved in the binding of NC-HK 1 to the hydrophobic resin. In contrast, as expected, this treatment effectively altered the elution profile (and hydrophobic character) of the rat enzyme in the column (Figure 3B, open squares) (27). This suggests that maize NCHK 1 does not have the domain for chymotrypsin cleavage present in rat HK (27).

In fact, the first $24 \mathrm{~N}$-terminal residues of the spinach chloroplast $S o H K 1$ protein deduced from the cDNA gene are extremely hydrophobic (13), conferring to this plant HK a longer and more hydrophobic N-terminal region than the one found in rat brain HK. Analysis by a hydropathy plot (39) of spinach and rice HK cDNA genes ( $\mathrm{SoHK} 1$ and $O s \mathrm{HK} 1$, respectively) and a putative maize NC-HK ( $\mathrm{ZmHK} 1)$ cDNA gene revealed the presence of a similar hydrophobic $\mathrm{N}$-terminal sequence in these enzymes (Figure 4). The rice NC-HK (OsHK 1$)$ is kineti-

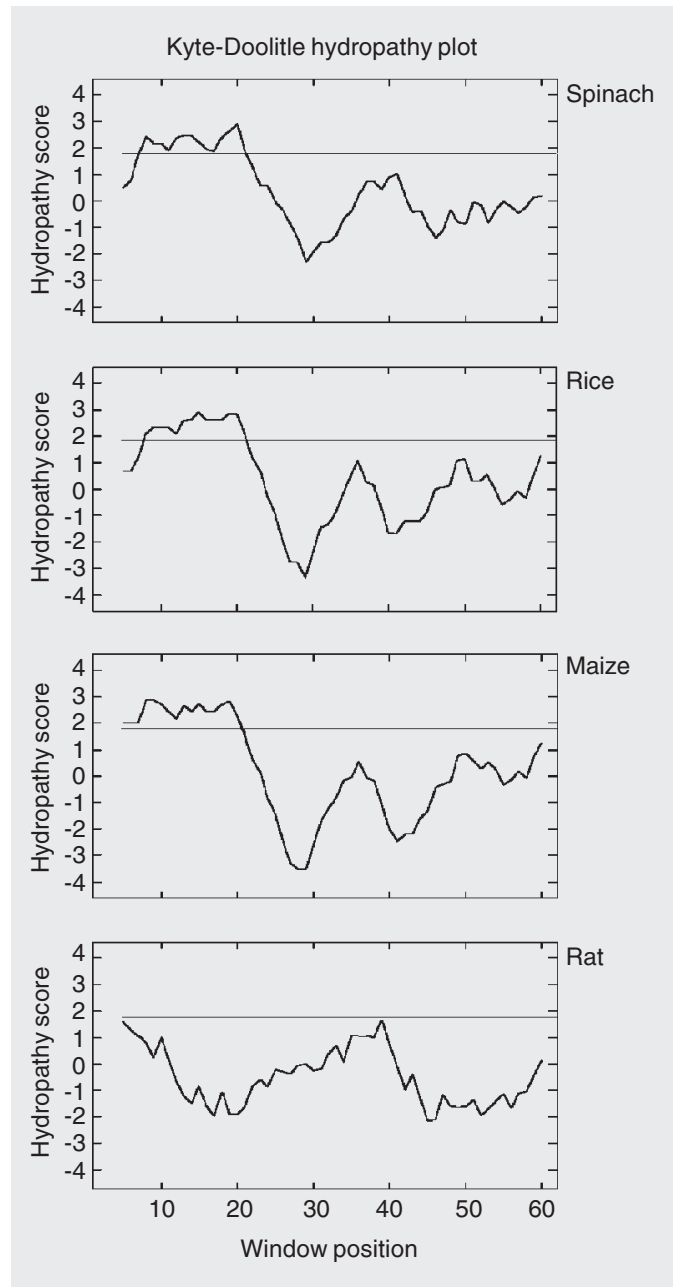

Figure 4. Kyte-Doolittle hydropathy plot of the first $60 \mathrm{~N}$ terminal amino acids of membrane-bound hexokinases found in spinach, rice, maize, and rat brain. The thick horizontal line shows the hydropathy score limit at which the sequence presented a highly hydrophobic character. In plants the first 20 amino acids are mostly above the line. 
cally very closely related to the NC-HK found in maize roots $(11,14,28)$ and it is not solubilized by glucose-6-phosphate, clotrimazole or thiopental treatment in a similar manner as that observed for maize NC-HK. One possibility is that maize NC-HK and rice OsHK 1 cDNA genes are homologous and have the same anchor hydrophobic domain.

The prediction of transmembrane helices was made using the program TMHMM, version 2.0, at www.cbs.dtu.dk/services/ TMHMM/. The HKs from tobacco and spinach (NtHK 1 and SoHK 1) that are located on the outer envelope membrane of chloroplasts (13) and the HKs from Arabidopsis and rice (At HK 1 and $O s \mathrm{HK} 1$ ), that were found to be associated with the mitochondrial membrane $(18,28)$, possess a transmembrane helix domain, allowing them to be directly immersed into the membrane. There is an HK cDNA gene in maize $(Z m H K$ 1) whose deduced protein also has a transmembrane helix. Therefore, it may be possible to suggest that the maize NC-HK proteins studied here belong to this group of membrane-bound plant HKs. However, we cannot exclude the possibility that maize NC-HK is interacting with porin in a manner that is insensitive to solubilization via glucose-6-phosphate, clotrimazole or thiopental. Our data provide experimental evidence (23) that HK from plants is unable to bind to mitochondria in the same manner as mammalian HK. Further studies are needed to evaluate how the tight association of the NC-HK with the mitochondrial outer membrane may interfere with the properties of apoptosis and reactive oxygen species generation (40) in plants.

\section{References}

1. Saltman P. Hexokinase in higher plants. J Biol Chem 1953; 200: 145-154.

2. Marré E, Cornaggia MP, Bianchetti R. The effects of sugars on the development of hexose phosphorylating enzymes in the castor bean cotyledons. Phytochemistry 1968; 7: 1115-1123.

3. Baldus B, Kelly GJ, Latzko E. Hexokinases of spinach leaves. Phytochemistry 1981; 20: 1811-1814.

4. Krook J, Vreugdenhil D, van der Plas LHW. Uptake and phosphorylation of glucose and fructose in Dacus carota cell suspension are differently regulated. Plant Physiol Biochem 2000; 38: 603-612.

5. Baijal M, Sanwal GG. Intracellular localization of hexokinase in Cuscuta reflexa. Phytochemistry 1977; 16: 329-332.

6. Miernyk JA, Dennis DT. Mitochondrial, plastid, and cytosolic isozymes of hexokinase from developing endosperm of Ricinus communis. Arch Biochem Biophys 1983; 226: 458-468.

7. Cosio E, Bustamante E. Subcellular localization of hexokinase in pea leaves. Evidence for the predominance of a mitochondrially bound form. J Biol Chem 1984; 259: 7688-7692.

8. Baysdorfer C, Kremer DF, Sicher RC. Partial purification of fructokinase activity from barley leaves. J Plant Physiol 1989; 134: 156-161.

9. Schnarrenberger C. Characterization and compartmentation, in green leaves, of hexokinases with different specificities for glucose, fructose, and mannose and for nucleoside triphosphates. Planta 1990; 181: 249-255.

10. Stewart DC, Copeland L. Mitochondrial hexokinase of avocado mesocarp. Phytochemistry 1993; 32: 27-30.

11. Galina A, Reis M, Albuquerque MC, Puyou AG, Puyou MT, de Meis L. Different properties of the mitochondrial and cytosolic hexokinases in maize roots. Biochem J 1995; 309 (Pt 1): 105-112.

12. Singh KK, Chen C, Epstein DK, Gibbs M. Respiration of sugars in spinach (Spinacia oleracea), maize (Zea mays), and Chlamydomonas reinhardtii F-60 chloroplasts with emphasis on the hexose kinases. Plant Physiol 1993; 102: 587-593.

13. Wiese A, Groner F, Sonnewald U, Deppner H, Lerchl J, Hebbeker U, et al. Spinach hexokinase I is located in the outer envelope membrane of plastids. FEBS Lett 1999; 461: 13-18.

14. da-Silva WS, Rezende GL, Galina A. Subcellular distribution and kinetic properties of cytosolic and non-cytosolic hexokinases in maize seedling roots: implications for hexose phosphorylation. $J$ Exp Bot 2001; 52: 1191-1201.

15. Jang JC, Sheen J. Sugar sensing in higher plants. Plant Cell 1994; 6: 1665-1679.

16. Umemura T, Perata $P$, Futsuhara $Y$, Yamaguchi J. Sugar sensing and alpha-amylase gene repression in rice embryos. Planta 1998; 204: 420-428.

17. Galina A, da-Silva WS. Hexokinase activity alters sugar-nucleotide formation in maize root homogenates. Phytochemistry 2000; 53: 2937.

18. Rolland F, Moore B, Sheen J. Sugar sensing and signaling in plants. Plant Cell 2002; 14 (Suppl): S185-S205.

19. Moore B, Zhou L, Rolland F, Hall Q, Cheng WH, Liu YX, et al. Role of the Arabidopsis glucose sensor HXK1 in nutrient, light, and hormonal signaling. Science 2003; 300: 332-336.

20. Wilson JE. Brain hexokinase. A proposed relation between solubleparticulate distribution and activity in vivo. J Biol Chem 1968; 243: 3640-3647.

21. Adams V, Griffin L, Towbin J, Gelb B, Worley K, McCabe ER. Porin interaction with hexokinase and glycerol kinase: metabolic microcompartmentation at the outer mitochondrial membrane. Biochem Med Metab Biol 1991; 45: 271-291.

22. Kabir F, Wilson JE. Mitochondrial hexokinase in brain of various 
species: differences in sensitivity to solubilization by glucose 6phosphate. Arch Biochem Biophys 1993; 300: 641-650.

23. Wilson JE. Homologous and heterologous interactions between hexokinase and mitochondrial porin: evolutionary implications. $J$ Bioenerg Biomembr 1997; 29: 97-102.

24. Hashimoto M, Wilson JE. Membrane potential-dependent conformational changes in mitochondrially bound hexokinase of brain. Arch Biochem Biophys 2000; 384: 163-173.

25. Krieglstein J, Schachtschabel DO, Wever K, Wickop G. Influence of thiopental on intracellular distribution of hexokinase activity in various tumor cells. Arzneimittelforschung 1981; 31: 121-123.

26. Penso J, Beitner R. Clotrimazole and bifonazole detach hexokinase from mitochondria of melanoma cells. Eur J Pharmacol 1998; 342: 113-117.

27. Kurokawa M, Yokoyama K, Kaneko M, Ishibashi S. Difference in hydrophobicity between mitochondria-bindable and non-bindable forms of hexokinase purified from rat brain. Biochem Biophys Res Commun 1983; 115: 1101-1107.

28. Sunako T, Morita A, Yamaguchi J. Subcellular localization of rice hexosekinase, OsHxK1. Rice Genet Newsl 1999; 16: 120-122.

29. Galina A, Logullo C, Souza EF, Rezende GL, da-Silva W. Sugar phosphorylation modulates ADP inhibition of maize mitochondrial hexokinase. Physiol Plant 1999; 105: 17-23.

30. Lowry OH, Rosebrough NJ, Farr AL, Randall RJ. Protein measurement with the Folin phenol reagent. J Biol Chem 1951; 193: 265275.

31. Hjelmeland LM. A nondenaturing zwitterionic detergent for membrane biochemistry: design and synthesis. Proc Natl Acad Sci USA 1980; 77: 6368-6370.
32. Hjelmeland LM, Chrambach A. Solubilization of functional membrane proteins. Methods Enzymol 1984; 104: 305-318.

33. Doehlert DC. Separation and characterization of four hexose kinases from developing maize kernels. Plant Physiol 1989; 89: 10421048.

34. Gardner A, Davies HV, Burch LR. Purification and properties of fructokinase from developing tubers of potato (Solanum tuberosum L.). Plant Physiol 1992; 100: 178-183.

35. Renz A, Merlo L, Stitt M. Partial purification from potato tubers of three fructokinases and three hexokinases which show differing organ and developmental specificity. Planta 1993; 190: 156-165.

36. Martinez-Barajas E, Randall DD. Purification and characterization of a glucokinase from young tomato (Lycopersicon esculentum L. Mill.) fruit. Planta 1998; 205: 567-573.

37. Yamamoto YT, Prata RTN, Williamson JD, Weddington M, Pharr DM. Formation of a hexokinase complex is associated with changes in energy utilization in celery organ and cells. Physiol Plant 2000; 110: 28-37.

38. Cardenas ML, Cornish-Bowden A, Ureta T. Evolution and regulatory role of the hexokinases. Biochim Biophys Acta 1998; 1401: 242 264.

39. Kyte J, Doolittle RF. A simple method for displaying the hydropathic character of a protein. J Mol Biol 1982; 157: 105-132.

40. da-Silva WS, Gomez-Puyou A, de Gomez-Puyou MT, MorenoSanchez R, De Felice FG, de Meis L, et al. Mitochondrial bound hexokinase activity as a preventive antioxidant defense: steadystate ADP formation as a regulatory mechanism of membrane potential and reactive oxygen species generation in mitochondria. $J$ Biol Chem 2004; 279: 39846-39855. 